\title{
Oligometastatic Breast Cancer: A Shift from Palliative to Potentially Curative Treatment?
}

\author{
Simona Di Lascio ${ }^{a, b}$ Olivia Pagani ${ }^{a}, b$ \\ anstitute of Oncology of Southern Switzerland (IOSI), 'breast Unit of Southern Switzerland (CSSI), Lugano, Switzerland
}

\author{
Keywords \\ Breast cancer · Oligometastatic disease $\cdot$ Treatment $\cdot$ Multi- \\ disciplinary team
}

\section{Summary}

A distinctive subset of metastatic breast cancer is represented by the so called 'oligometastatic' disease, characterized by single/few detectable metastatic lesions. A more aggressive multidisciplinary approach can be considered in this patient population: available data report favorable results of 'radical' local therapy for limited metastatic disease at least in a subset of selected patients. Selection bias and the retrospective nature of data do not allow for generalization of the results: the use of such approaches must be individualized and managed within a multidisciplinary team of dedicated specialists. Improvement in surgical and radiation techniques, development of new tools to deliver local chemotherapy, and new procedures (i.e. cryosurgery, laser and microwave ablation) mandate careful evaluation of such single and combined modalities in controlled clinical trials. A more accurate identification of patients with limited metastases and better definition of treatment endpoints will also allow correct patient selection for locally aggressive therapies. This paper focusses on local treatment of the primary tumor and of the most frequent distant disease sites in the presence of oligometastatic disease.

\section{Introduction}

Despite breast cancer (BC) being the most common cancer in women in the developed world, only a minority of patients $(<10 \%)$ has stage IV disease at diagnosis [1]. In addition, 20$30 \%$ of patients with early $\mathrm{BC}$ will experience distant metastatic relapse [2]. Due to advances in available multimodality therapies and a better understanding of tumor biology, survival of stage IV patients is constantly improving [3-5]. The oligometastatic (OM) state implies that a few metastases (usually $\leq 5$ ) exist before tumor cells acquire widespread metastatic potential [6]. Some tumor cell characteristics (altered cell adhesion, intravasation, and bloodstream survival) seem to favor the metastatic spread [7], while others such as tumor dormancy could result in limited dissemination [8]. OM phenotypes have also been recently identified from various tumor types and metastatic sites, showing different genetic signatures between patients with few or many metastases [9]. A current refinement is the concept of oligorecurrence, i.e. patients with a limited number of metastases and controlled primary tumors [10]. Clinically, OM BC is characterized by solitary/few detectable lesions, usually limited to single organs, in which local therapy with curative intent could impact survival. This population of 'potentially curable' stage IV disease is estimated to be $1-10 \%$ of newly diagnosed patients with metastatic BC (MBC) [5]. A multimodal approach is endorsed for these selected patients $[5,11]$. The identification of patients with truly OM disease is challenging. Most published series refer to an era before modern imaging (i.e. positron emission tomography-computed tomography), and thus many patients were probably under-staged, potentially leading to underestimation of the global effect of an aggressive local management. A recent series in stage I-III BC found $16 \%$ of patients with OM disease (mean of 1.7 lesions/patient), with a higher proportion in asymptomatic patients [12]. Improved imaging may also prevent aggressive approaches to be employed in patients with only apparently limited disease [13]. Patients with OM disease can be divided into 3 cohorts [10, 14]: i) those who present with oligometastases; ii) those with residual oligometastases after systemic therapy (ST); and iii) those with relapsed oligometastases after curative locoregional therapy. These different groups have possibly distinct prognoses, and may need differential approaches. To this extent, a broader staging system encompassing solitary metastases and oligometastases could be of help [15]. Local treatment of OM BC is distinct from common indications of radiotherapy (RT) and surgery in MBC, applied as consolidation of ST responses or debulking strategies. This review will summarize the available therapeutic options possibly associated with cure in $\mathrm{OM} \mathrm{BC}$, focusing on local management of the primary tumor and multimodal local treatments of oligometastases to the liver, lung, bone, and brain.

\begin{tabular}{ll}
\hline KARGER & $\begin{array}{l}\text { (1) 2014 S. Karger GmbH, Freiburg } \\
\text { 1661-3791/14/0091-0007\$39.50/0 }\end{array}$ \\
$\begin{array}{l}\text { Fax +497614520714 } \\
\text { Information@Karger.com } \\
\text { www.karger.com }\end{array}$ & $\begin{array}{l}\text { Accessible online at: } \\
\text { www.karger.com/brc }\end{array}$
\end{tabular}

Dr. Simona Di Lascio 


\section{Locoregional Treatment of the Primary}

Retrospective series of thousands of patients consistently show an overall survival (OS) advantage (pooled hazard ratio (HR) 0.65-0.70) from 'radical' management of the primary tumor, either by surgery $[16,17]$ or definitive RT $[18,19]$, in patients presenting with MBC. Possible explanations include: i) patient selection bias (i.e. lower disease burden, better general condition, younger age); ii) restoration of immunocompetence by removal of immunosuppressive factors [20]; iii) decreased tumor burden in the body; iv) removal of the 'seed' source of new metastases [21]; v) decrease in potentially resistant cell lines [4]; and vi) increased chemosensitivity due to angiogenesis in distant disease sites instigated by surgery. It is not clear which patients most likely benefit from treatment of the primary tumor: those with estrogen-responsive tumors, bone metastases (BoM) only, low disease burden at diagnosis, or those who respond to ST?

Some potential disadvantages of surgery have arisen; with the primary tumor being a source of antiangiogenic factors and growth factor inhibitors, an accelerated relapse might follow its removal [22]. The possible release of growth factors related to surgical wounding [23] and the immunosuppression caused by surgery and anesthesia [24] are also potential drawbacks. At the same time, improvements in surgery and anesthesia have led to a significant reduction in morbidity, resulting in more patients being able to benefit from this approach.

The population-based databases $[17,25,26]$ include large numbers of patients but suffer from lack of details (i.e. hormone receptor (HR) status, details of any RT, margin status) which have shown to be significant in multivariate analyses. Single institute series, in contrast, allow an in depth analysis of various prognostic and confounding factors but are limited by the small number of patients and institutional biases in patient selection. Unresolved locoregional questions from retrospective series include the timing (upfront or after response to ST) and the extent of surgery (i.e. need of free margins and benefit of axillary dissection (AD)) and the role of 'adjuvant' RT [27]. Large databases [17, 25] capture the first treatment, and therefore patients who had surgery most likely had it upfront. Regional RT data are limited to the study of Rapiti et al. [26] who reported an increased hazard of death in women who did not receive RT after breast-conserving surgery (BCS).

A recent meta-analysis of 28,693 MBC patients from 10 studies $(52.8 \%$ with surgery of the primary) showed a superior 3-year survival for resected patients (odds ratio (OR) $2.32,95 \%$ confidence interval (CI) 2.08-2.6; $\mathrm{p}<0.01$ ); of note, $22 \%$ of patients who received ST alone were alive compared to $40 \%$ of those who underwent breast surgery [28]. Subgroup analyses favored smaller tumors, less competing medical comorbidities, and lower distant disease burden $(\mathrm{p}<0.01)$. Conversely, there was no statistical difference between the 2 groups according to metastatic sites, tumor grade, or HR status. Data for the type of surgery were available for 14,488 patients (61\% mastectomy, $39 \%$ BCS): 1 of the analyzed studies comparing the 2 procedures found no difference in outcome [29]. With regard to the axilla $(n=486), 93 \%$ had AD and $7 \%$ had sentinel node biopsy. 2 studies found no OS improvement in patients undergoing $\mathrm{AD}[25,29]$, while another showed reduced risk of death with AD in patients with negative margins, not achieving statistical significance [26]. Margins ( $n=5,791)$ were positive in $43 \%$ of patients; 2 studies demonstrated that patients with negative margins had the longest survival $[25,26]$. Main limitations of this analysis were its retrospective nature and patient selection bias. In addition, data on HER2+ patients are missing in most studies, limiting the applicability of these results in all disease subgroups and in the modern era of HER2-targeted therapies.

Five randomized, controlled trials in the US/India/Austria/ Netherlands/Turkey address the role of primary tumor excision in MBC. The results of the Indian and Turkish trials were recently presented and showed no statistically significant difference in survival between patients undergoing surgery versus those receiving ST [30, 31]. In the Indian study $(\mathrm{n}=350)$ [30], patients were randomized after 6 months of anthracyline/ taxane-based chemotherapy and were stratified according to site/number of distant metastases and HR status. Surgery consisted of BCS or modified radical mastectomy $+\mathrm{AD} \pm$ supraclavicular clearance if indicated. All patients with HR-positive disease received endocrine therapy. The lack of survival benefit was mainly driven by a tradeoff between local and distant control with an apparent growth advantage on distant metastases in patients randomized to local treatment: no difference in any subgroup was evident. In the Turkish trial $(\mathrm{n}=278)$ [31], patients were randomized upfront, no stratification was planned. AD was performed only in patients with positive nodes, free margins were mandatory, and RT was given after BCS. Endocrine therapy and trastuzumab were given in all patients if indicated, local therapy to distant metastases and bisphosphonates according to the investigator's choice. A trend in improved survival was shown in patients with bone-only disease, limited metastatic burden, and favorable histology.

Longer follow-up of these trials is needed as well as results from the other randomized trials with different designs (i.e. randomization only in patients responding to $\mathrm{ST}$ ) to clarify the role, if any, of breast treatment in $\operatorname{MBC}[32,33]$. In summary, there is a bulk of retrospective data suggesting a positive impact of local treatment of the primary tumor in MBC. While waiting for definitive data from the randomized studies, surgery for primary breast tumors can be considered as a cheap and low-morbidity treatment which can offer rapid local control and potentially a survival benefit, provided it is performed optimally. As ST improve and survival time increases, uncontrolled local disease will possibly become a concern, increasing the number of patients potentially eligible for breast surgery or exclusive locoregional RT. 


\section{Stereotactic Body Radiotherapy}

The role of RT in metastatic patients has recently evolved from palliative to potentially curative for selected OM disease. A variety of retrospective data suggest the addition of RT to metastatic sites may be associated with better survival [34, 35]. Three-dimensional conformal RT is considered standard in patients with OM disease: it allows an increase in the tumor dose and a reduction in normal tissue toxicity by limiting the RT field only to the metastatic lesions, potentially allowing safe delivery of curative doses even concomitantly to ST. In the last decade, different forms of highly conformal RT (stereotactic body radiotherapy (SBRT), image-guided RT (IGRT), radiosurgery) have emerged as potentially curative therapeutic strategies for localized lesions. The American Society of Radiation Oncology defines SBRT as external beam RT delivering with high precision a high dose of RT to an extracranial target as a single dose or a small number of fractions [36]. A wide range of techniques, doses, and dose fractionation schedules can be delivered: as SBRT fractionation schemes have been developed empirically, the optimal fraction size/number has still to be defined [35]. Most commonly, SBRT consists of 1-5 fractions of 10-20 Gy (as compared to 2 Gy/day over 6-8 weeks), with a high dose in the middle of the tumor and a sharp decrease at the edge, which prevents effective dose delivery in tumors $>4-5 \mathrm{~cm}$ in diameter. Given the impossibility to increase the target volume, it is important to control target motility by either motion restriction, target tracking, or gating. The biological effect of SBRT may be different from conventional fractionated RT: in addition to the direct cell kill within the high-dose region, vascular and stromal effects likely contribute to better tumor control [37]. The higher dose (as much as $30 \%$ in the center of the target volume) could also be more effective against hypoxic or tumor stem cells and might deprive the rest of the tumor of pro-growth and prosurvival factors [38]. Activation of innate and adaptive immune responses have also been proposed as important host factors for tumor control [39]. Published studies of SBRT for metastatic disease included: i) various types of primary tumors and metastatic sites; ii) a single metastatic site (i.e. lung or liver); or iii) a specific tumor type. These different scenarios can be important determinants of outcome, as some histological types or disease sites are possibly more responsive to radical treatment than others [40]. Overall, SBRT of limited metastases has shown promising local control rates ranging from 67 to $95 \%$ [34, 35, 39], and compares favorably with surgery in terms of results and tolerability. In addition, SBRT may be more broadly applicable to greater numbers of sites in different organs. When results are analyzed according to tumor type, patients with $\mathrm{BC}$ have a much better survival as compared to other cancers (mainly colorectal or lung) with 36 versus $13 \%$ progression-free survival (PFS) at 2 years and 47 versus 9\% OS at 6 years. The rate of local control is also higher ( 87 vs. $74 \%$ ), suggesting that better local management might result in improved PFS [40]. When specific disease sites are considered [34, 35], the 2 -year local control rate is approximately $80 \%$ for lung metastases (LuM), with a corresponding 2-year survival of $50 \%$, and a $5 \%$ rate of grade $\geq 3$ toxicities. The 2 -year local control rate varies between 57 and $92 \%$ for liver metastases (LiM), and radiation-induced liver damage is exceptional. SBRT is also particularly interesting for spinal and brain metastases $(\mathrm{BrM})$ and allows for re-irradiations. Most of the reported data are retrospective, but at least 4 prospective phase II trials and 2 randomized studies (none of them restricted to BC) are ongoing [39]. In summary, association of ST and SBRT may be used for optimized control of OM disease. A better definition of target volumes/doses, patient eligibility, and place of SBRT in the global therapeutic strategy of OM BC is warranted.

\section{Local Treatment of Selected Disease Sites}

When discussing local strategies for $\mathrm{OM} \mathrm{BC}$, the recent therapeutic developments of ST (i.e. the introduction of new cytotoxic, endocrine, and biologic therapies), which have resulted in constant improvements in treatment efficacy and MBC outcome, should always be taken into account [4]. The long-term follow-up of a large population-based series from British Columbia $(n=2,150)$ [41] and of 1,581 consecutive first-line patients treated at the MD Anderson Cancer Center (MDACC) [42] shows in both series a median survival of approximately 22 months, with $16.6 \%$ patients achieving a complete response $(\mathrm{CR})$ in the MDACC series which resulted in an extended median survival of 41.8 months. These results with modern ST have always to be considered and compared to the outcome of local strategies in OM BC.

\section{Liver}

Along with bone and lung, liver is a common MBC site, but only $4-5 \%$ of patients have isolated liver involvement. Overall, patients with LiM have a median survival of 4-33 months, and treatment options are usually restricted to palliative ST [43]. When evaluating outcome according to different subtypes and clinical features, triple negative (TN) disease, time to LiM $<24$ months, and $\geq 3$ lesions are significant predictors of poor survival [44]. With modern ST, the median survival of patients with LiM alone can exceed 2 years, but most patients do ultimately progress in the liver, suggesting a more aggressive multimodality treatment through prospective clinical trials is worth exploring in this specific subset of patients [45]. Patients with isolated LiM can potentially be managed with local treatments (surgery, radiofrequency ablation (RFA), transcatheter arterial chemoembolization (TACE)). In contrast to liver colorectal metastases, local treatment for BC 
LiM is not considered a therapeutic option due to common involvement of additional organs; nevertheless, in some selected patients, local approaches have been associated with long-term survival [46, 47].

\section{Surgery}

Most series have reported prolonged OS with liver metastasectomy in patients with a disease-free interval (DFI) of $>2$ years, solitary metastasis, good response to chemotherapy, and negative surgical margins [43, 48]. Recently, systematic literature reviews, mostly of small selected groups of patients, have shown comparable outcomes to colorectal liver disease with 5-year survival rates ranging between 20 and 60\% [46, 47]. Additional favorable independent prognostic factors are: absence of extrahepatic disease (except for isolated lung and bony metastasis); at least a partial response to ST before surgery; endocrine responsive disease [48].

\section{Transcatheter Arterial Chemoembolization}

TACE delivers high-dose chemotherapy directly to the LiM and can be offered to patients with unresectable LiM. Approximately $80 \%$ of the blood supply to LiM is derived from the hepatic artery, and direct tumor infusions, compared with ST, result in higher drug concentrations, potentially overcoming tumor resistance. In addition, direct liver delivery may result in a better systemic toxicity profile. The chemotherapeutic effect of TACE on tumor cells is also increased by embolization-induced tumor ischemia [49]. Published data suggest that TACE may have a palliative role in delaying progression of isolated LiM. There is currently only scarce data regarding its role in the management of $\mathrm{MBC}$, but tumor shrinkage can be dramatic and may render patients candidates for liver resection with curative intent, potentially resulting in long-term disease-free survival [50]. In the published series, TACE commonly follows progression under ST: a recent study compared combined TACE and ST for liveronly metastases $(n=44)$ to ST alone $(n=43)$. The 3-year survival rate was $47.6 \%$ in the combined treatment group versus $7.4 \%$ in the ST alone group ( $\mathrm{p}=0.027)$. HR-negative status of the primary tumor and DFI from diagnosis to LiM $<24$ months were independently associated with poor prognosis [51]. Unresolved questions include the benefit of using multiple chemotherapeutic drugs as opposed to single- or twoagent TACE, the role of repeated cycles, and its sequence in the therapeutic algorithm.

\section{Radiofrequency Ablation}

RFA uses low frequency/long wavelength radiowaves to generate heat within a tumor mass causing thermal coagulative necrosis. RFA can be performed via an open, laparoscopic, or image-guided percutaneous approach under general or local anesthesia. RFA is a relatively safe procedure, with mortality between 0.3 and $0.8 \%$ and morbidity ranging from 2 to $10 \%$. Effective local control can be achieved mostly in solitary lesions $<3 \mathrm{~cm}$ in diameter [52]. The majority of data regarding LiM come from single-arm, retrospective or prospective series in unresectable colorectal metastases. In BC, the reported data show a median survival of 30-60 months, with no treatment-related deaths and only 3 serious treatment-related adverse events reported [53]. A recent study compared laparoscopic RFA in BC patients who failed to respond or showed an incomplete response to ST $(n=24)$ or to ST alone $(n=32)$ : patients were matched by size and number of LiM, and the 2 groups were similar in HR status and chemotherapy exposure. OS after the diagnosis of LiM was 47 months in the RFA group and 9 months in the ST-only group ( $p=0.0001)$ [54]. Skepticism remains over the efficacy of RFA in BC due to the heterogeneity of patient inclusion and selective nature of reporting. Randomized control trials directly comparing RFA to liver resection versus best ST alone versus their combination should ideally be conducted. Additional low-toxicity local procedures include cryosurgery, laser-induced interstitial thermotherapy, microwave ablation, high-intensity focused ultrasound (HIFU), or tumor embolization with isotope-loaded microspheres. Encouraging results have been reported which need confirmatory data in comparison or addition to ST or other local approaches $[4,55,56]$.

\section{Summary}

Local treatment of BC LiM can be discussed in selected patients with $\mathrm{OM}$ disease, taking into account several factors such as tumor subtype, time to LiM, number/size of lesions and response to previous ST, to avoid unnecessary morbidity and ensure a potential survival benefit for selected patients.

\section{Lung}

While resection of LuM is a common treatment in other tumors, the role in $\mathrm{BC}$ is still unclear [57]. The reported data refer to highly selected patients, and any comparison with surgically untreated patients is threatened by serious biases. Lung resection in $\mathrm{MBC}$ is also an important diagnostic tool: the proportion of lesions proved not to be $\mathrm{BC}$ in various series ranges from 7 to $66 \%$ [5]. In the case of biopsy-proven $\mathrm{BC} \mathrm{LuM}$, the level of evidence for curative resection is low, especially when comparing survival rates after complete resection and after CR to modern ST. Open surgery, as opposed to thoracoscopic surgery should be preferred to exclude invisible nodules at diagnostic imaging. Discussed favorable prognostic factors for lung resection include: DFI > 36 months, solitary metastasis, HR positivity, small size of metastases, and complete resection [5], but not all published series show different outcomes according to these features, in particular the number of lesions and HR status [58]. Long DFI is usually associated with slow-growing tumors, long effective endocrine treatment, and less tumor burden. A historical series of patients with BC lung-only metastases treated with chemo- 
therapy alone at MDACC showed a median OS of 22 months and a 5-year survival of $16 \%$ [59]. Patients with limited disease (1-2 lesions $\leq 2 \mathrm{~cm}$ diameter) achieved a median OS of 57 months, which is almost identical to the historical surgical data of Friedel et al. [60] showing a median survival of 59 months in the best prognostic group (DFI $>3$ years and a single completely resected metastasis). On the other hand, only $33 \%$ of the patients with an isolated LuM achieved a CR under chemotherapy, which resulted in a median OS of 37 months [59]. In a recent German retrospective series $(\mathrm{n}=47)$, a median OS of 32 months and a 5-year survival of $36 \%$ was shown [61]. Number of metastases, complete versus incomplete resection, and nodal/pleural/chest wall involvement did not impact survival, as opposed to HR (5-year survival rates of 76 and $12 \%$ for positive and negative HR, respectively; $p=0.002$ ) and HER2 status ( 92 and $62.5 \%$ for positive and negative HER2, respectively; $p=0.037$ ). Most patients received 'adjuvant' ST after surgery, including endocrine and targeted agents. A prospective, recent German series $(n=81)$ showed that $\mathrm{R} 0$ resection (achieved in $81.5 \%$ of patients) was associated with a significantly longer median OS than R1/R2 resections (103.4 vs. 23.6 vs. 20.2 months, respectively; $\mathrm{p}<0.001)$. Multivariate analysis revealed $\mathrm{R} 0$ resection, number $(\geq 2)$, size $(\geq 3 \mathrm{~cm})$, and $\mathrm{HR}$ positivity of metastases as independent prognostic factors for long-term survival [62]. In summary, as the morbidity and mortality of lung resection has decreased significantly over the last decades, this potentially beneficial procedure can be discussed in selected patients. Before proceeding with surgery, potential tumor control by ST, safety and feasibility of surgery, tumor biology, number of lesions, and DFI must be evaluated by a multidisciplinary dedicated team.

\section{Bone}

Bone is the third most common site of BC metastases. At $\mathrm{BC}$ diagnosis, 5-6\% of patients present with distant spread, bone being the most common metastatic site. Bone is also the first site of distant relapse in $>50 \%$ of patients, and bone-only metastases occur in $17-37 \%$ of patients with distant relapses. Although any bone may be affected, extremities are less involved than other sites. $25 \%$ of $\mathrm{BC}$ patients with BoM are asymptomatic and diagnosed by chance; in the remaining $75 \%$, BoM are associated with skeletal-related events (SRE) [63]. Magnetic resonance imaging (MRI) is the most sensitive imaging modality for detection of BoM, especially for vertebral metastases and when bone scintigraphy is equivocal, and can add important information when local treatment is discussed [64]. Patients with HR positivity, low-intermediate histologic grade, and low mitotic rate tend to metastasize more to bone than to viscera [65]. A short DFI ( $<2$ years) has been associated with worse prognosis and increased mortality [66] in patients with bone-only metastases. In a retrospective study on $110 \mathrm{BC}$ patients with bone-only metastases, multivariate analyses showed that solitary BoM, HR positivity, and bisphosphonate treatment were significantly associated with prolonged OS [67]. The median OS in patients with bone-only disease is about 26 months, as opposed to 21 months in patients with bone and visceral metastases and 18 months in patients with visceral metastases only [68]. An additional favorable prognostic factor is the number of BoM, with improved survival for solitary compared to multiple lesions [69]. A solitary BoM can often be successfully treated, and longlasting complete remission is not unusual.

\section{Local Treatment of Solitary BoM}

Surgery is indicated in most cases of pathological fractures. In a population-based study, Wedin et al. [70] found that $17 \%$ of $\mathrm{BC}$ patients who presented with symptomatic BoM needed surgery. Survival prognostic factors after surgery have been evaluated in a series of 115 consecutive patients [71]: in multivariate analysis, patients with a solitary BoM had the best survival (median of 65 months) as compared to patients with visceral disease (median of 13 months), with no impact of age and extent of surgery. RT remains a keystone therapy for the treatment of BoM, in particular of spinal lesions. Pain relief has been the historical goal of conventional RT, but improvements in ST with long-term control of MBC, increased diagnostic possibility of solitary BoM, and availability of SBRT are changing the role of RT in the management of these patients. Patients with BoM treated with SBRT have better survival and tend to have a better PFS than patients with LuM or LiM [72]. Multiple additional conservative approaches are available for BoM; despite no proven survival benefit, isolated BoM are commonly treated with vertebroplasty (PV) and kyphoplasty (PK) with the aim of delaying BoM-related morbidity and maintaining/improving quality of life (QoL). In percutaneous computed tomographic fluoroscopy-guided PV, bone cement is injected through a minimal incision into the fractured site. In PK, a balloon is inserted into the fractured site and inflated-deflated to create a cavity: the balloon is then taken out prior to cement injection. Both procedures are superior to oral pain management: no direct comparison is available but systematic reviews of the existing data showed very little difference in clinical outcomes of the 2 techniques [73]. The overall rate of complications associated with PV and PK is low, but the rate of cement extravasation is higher in $\mathrm{PV}$. PV of painful osteolytic spinal metastases from BC is safe even in the case of substantial involvement of the vertebral cross-sectional area or cortical bone $[73,74]$. To establish the relative strengths and weaknesses of all these procedures, well-designed randomized clinical trials are required.

\section{Summary}

Considering the number of current treatments available for BoM and different patients' prognosis, based on tumor biology and number of lesions, a multidisciplinary approach is 
mandatory to choose the best therapeutic strategy in the individual patient.

\section{Brain}

Approximately $10-15 \%$ of all MBC patients will develop symptomatic BrM with an increased risk and a shorter BrMfree survival in TN and HER2+ tumors compared with luminal or HER2- subtypes [75-77]. Survival of patients with BrM ranges from 2 to 16 months [78]. Brain-only metastases occur in about $17 \%$ of $\mathrm{BC}$ with $\mathrm{BrM}$, regardless of histologic subtype: this subset of patients with isolated BrM has a much more favorable outcome, and long-term survival is not uncommon [79]. HER2+ patients treated with trastuzumabbased therapy after completion of local therapy for BrM (surgery, RT) have significantly longer OS after diagnosis of BrM (4 vs. 14 months) [77]. Screening for BrM is not recommended as part of routine follow-up, as no evident benefit from early detection exists [11]. QoL and prevention of neurologic toxicity are important goals of care in this population. Apart from TN or HER2+ disease, established risk factors for the development of BrM are young age at BC diagnosis, presence of lung metastases, and short DFI [78]. The number of BrM is also an important prognostic factor for survival as well as for treatment selection [80].

Surgery or stereotactic radiosurgery (SRS), possibly integrated with whole brain RT (WBRT), are available options for patients with OM BrM, defined as 1-3 lesions. SRS is a single outpatient procedure associated with minimal acute toxicity. Most studies have not specifically addressed different tumor types despite the fact that management of extracranial disease differs widely based on histology. In particular, BC is not well represented in published phase III randomized studies of SRS (6.8-11.7\% of the study populations) [81]. TN subtype and progressive extracranial disease could be important adverse prognostic factors for OS after SRS [82]. Future studies specifically addressing treatment outcomes for well-defined patient groups and different tumor types are needed [83, 84]. A systematic review of the literature, comparing surgery, WBRT, single-dose SRS, and their combination [85-88] showed that: i) for patients with good performance status (PS), level 1 evidence exists that single-dose SRS+WBRT provides significantly superior local control compared with WBRT alone for patients with $\leq 4 \mathrm{BrM}$, without increased incidence of adverse events; ii) a significantly superior survival benefit is achieved by SRS+WBRT for patients with single $\mathrm{BrM}$, while for patients with $\geq 2 \mathrm{BrM}$ the survival advantage remains controversial [89]; iii) single-dose SRS alone provides equivalent survival compared to WBRT+single-dose SRS (level 2 evidence), with 1 randomized study in BC suggesting a survival advantage for the single-dose SRS alone strategy [90]; iv) single-dose SRS alone appears to be superior to WBRT alone for patients with $\leq 3 \mathrm{BrM}$ in terms of patient survival advantage; v) data are conflicting regarding the risk of recurrence at the treated area and progression at distant sites with single-dose SRS alone, suggesting further studies may be needed to define optimal dose prescription and/or dose rate for isolated SRS as opposed to SRS in the setting of an additive WBRT; vi) every $2-3$ months evaluation is warranted if single-dose SRS is utilized alone, for both efficacy and neurocognitive function assessment; vii) the potential differential neurocognitive consequences between SRS alone and SRS+WBRT have not been well studied and remain uncertain; viii) SRS+WBRT and resection+WBRT provide equivalence in survival (level 2 evidence); ix) weaker evidence (level 3) supports the use of SRS in lieu of resection+WBRT; $\mathrm{x})$ surgical resection+WBRT is superior to WBRT alone and to surgery alone in patients with good performance status (PS) and limited extracranial disease (level 1 evidence), with no significant differences in median survival, local control, or neurocognitive function between standard and altered dose/ fractionation schedules; and xi) larger lesions $(>3 \mathrm{~cm})$ or those causing significant mass effect $(1 \mathrm{~cm}$ midline shift) may have better outcomes with surgical resection than with SRS (level 2 evidence). Data on local RT, compared to WBRT, as well as on 2-3 dose versus single-dose SRS are not yet available in the setting of limited BrM. One treatment combination in need of further study is the concept of SRS to the surgical bed instead of postoperative WBRT: few retrospective case series suggest that both local control rates and survival are enhanced by this procedure but no robust prospective data yet exists. Current research is focused on the use of radiosensitizing and radioprotective strategies to improve the therapeutic ratio of WBRT [91]. Salvage SRS after WBRT for BC $\mathrm{BrM}$ is an additional promising tool, especially in patients with good PS [92, 93].

In summary, the management of OM BrM has significantly improved over the last decades, allowing for prolonged survival and potentially better QoL. Designing trials focusing on QoL and functional status as primary outcomes, rather than only recurrence or survival rates, should be a priority in this setting, given that small differences in survival rates between treatment options can be associated with significant differences in treatment-associated adverse events.

\section{Conclusion}

A more accurate identification of patients with truly OM disease will allow proper patient selection for locally aggressive therapies. Prospective and well-designed trials according to disease site and BC subtype might provide additional guidance for clinicians in the appropriate selection of the subset of patients for whom locally ablative therapies could impact OS while preserving adequate QoL. Delaying the need for systemic treatment/further treatment could also be of value, at least in some patients. Other outcome measures should be 
carefully evaluated in conjunction with survival, including symptoms (local and constitutional) and QoL, in view of the constantly prolonged survival of patients with $\mathrm{MBC}$ with modern systemic therapies. The role of 'adjuvant' medical or radiation treatments, as well the correct sequence and integration of all available procedures, should also be investigated to maximize the cost/benefit of these approaches. To achieve these scientific goals, global collaboration is needed: in the daily clinical practice, patients with OM BC should always be discussed within a multidisciplinary team of dedicated specialists to avoid on the one hand unnecessary aggressive therapies with limited proven efficacy but also to provide patients with the best chance of an increased QoL-oriented survival.

\section{Disclosure Statement}

The authors declare no conflicts of interest.

\section{References}

1 Howlader N, Noone AM, Krapcho M, et al. (eds): SEER cancer statistics review. Bethesda, MD, National Cancer Institute, 1975-2008. seer.cancer.gov/ csr/1975_2008/.

$\checkmark 2$ Early Breast Cancer Trialists' Collaborative Group: Effects of chemotherapy and hormonal therapy for early breast cancer on recurrence and 15-year survival: an overview of the randomised trials. Lancet 2005;365:1687-717.

3 Jemal A, Siegel R, Ward E, Murray T, Xu J, Thun MJ: Cancer statistics, 2007. CA Cancer J Clin 2007;57:43-66.

4 Senkus E, Cardoso F, Pagani O: Time for more optimism in metastatic breast cancer? Cancer Treat Rev 2014;40:220-8.

5 Pagani O, Senkus E, Wood W, et al.: International guidelines for management of metastatic breast cancer: can metastatic breast cancer be cured? J Natl Cancer Inst 2010;102:456-63.

6 Weichselbaum RR, Hellman S: Oligometastases revisited. Nat Rev Clin Oncol 2011;8:378-82.

7 Gupta GP, Massague J: Cancer metastasis: building a framework. Cell 2006;127:679-95.

$>8$ Willis L, Graham TA, Alarcón T, Alison MR, et al.: What can be learnt about disease progression in breast cancer dormancy from relapse data? PLoS One 2013;8:e62320.

-9 Lussier YA, Xing HR, Salama JK, et al.: MicroRNA expression characterizes oligometastasis(es). PLoS One 2011;6:e28650.

10 Niibe Y, Chang JY: Novel insights of oligometastases and oligo-recurrence and review of the literature. Pulm Med 2012;2012:261096.

11 Cardoso F, Costa A, Norton L, et al.: 1st International consensus guidelines for advanced breast cancer (ABC 1). Breast 2012;21:242-52.

12 Dorn P, Meriwether A, LeMieux M, et al.: Patterns of distant failure and progression in breast cancer: implications for the treatment of oligometastatic disease. Int J Radiat Oncol 2011;81:S643(abstr 2903).

13 Pennant M, Takwoingi Y, Pennant L, et al.: A systematic review of positron emission tomography (PET) and positron emission tomography/computed tomography $(\mathrm{PET} / \mathrm{CT})$ for the diagnosis of breast cancer recurrence. Health Technol Assess 2010;14:1-103.

14 Milano MT, Zhang H, Metcalfe SK, et al.: Oligometastatic breast cancer treated with curative-intent stereotactic body radiation therapy. Breast Cancer Res Treat 2009;115:601-8.

15 Rubin P, Brasacchio R, Katz A: Solitary metastases: illusion versus reality. Semin Radiat Oncol 2006;16:120-30.

16 Ruiterkamp J, Voogd AC, Bosscha K, TjanHeijnen VC, Ernst MF: Impact of breast surgery on survival in patients with distant metastases at initial presentation: a systematic review of the literature. Breast Cancer Res Treat 2010;120:9-16.

17 Gnerlich J, Dueker JM, Jeffe DB, et al.: Patient and tumor characteristics associated with primary tumor resection in women with stage IV breast cancer: analysis of 1988-2003 SEER data. Breast J 2008; $14: 538-42$.

18 Le Scodan SR, Stevens D, Brain E, et al.: Breast cancer with synchronous metastases: survival impact of exclusive locoregional radiotherapy. J Clin Oncol 2009;27:1375-81.

19 Ali D, Le Scodan R: Treatment of the primary tumor in breast cancer patients with synchronous metastases. Ann Oncol 2011;22:9-16.

20 Danna EA, Sinha P, Gilbert M, et al.: Surgical removal of primary tumor reverses tumor-induced immunosuppression despite the presence of metastatic disease. Cancer Res 2004;64:2205-11.

21 Morgan SC, Parker CC: Local treatment of metastatic cancer-killing the seed or disturbing the soil? Nat Rev Clin Oncol 2011;8:504-6.

22 Retsky MW, Demicheli R, Hrushesky WJ, et al.: Dormancy and surgery-driven escape from dormancy help explain some clinical features of breast cancer. APMIS 2008;116:730-41.

23 Babiera GV, Rao R, Feng L, et al.: Effect of primary tumor extirpation in breast cancer patients who present with stage IV disease and an intact primary tumor. Ann Surg Oncol 2006;13:776-82.

24 Salo M: Effects of anaesthesia and surgery on the immune response. Acta Anaesthesiol Scand 1992; 36:201-20.

25 Khan SA, Stewart AK, Morrow M: Does aggressive local therapy improve survival in metastatic breast cancer? Surgery 2002;132:620-7.

26 Rapiti E, Verkooijen HM, Vlastos G, et al.: Complete excision of primary breast tumor improves survival of patients with metastatic breast cancer at diagnosis. J Clin Oncol 2006;24:2743-9.

27 Deo SS, Jha D: Role of loco-regional surgery in metastatic breast cancer. J Can Res Ther 2013;9: 181-6.

28 Harris E, Barry M, Kell MR: Meta-analysis to determine if surgical resection of the primary tumour in the setting of stage IV breast cancer impacts on survival. Ann Surg Oncol 2013;20:2828-34.

29 Ruiterkamp J, Ernst MF, van de Poll-Franse LV, et al:: Surgical resection of the primary tumour is associated with improved survival in patients with distant metastatic breast cancer at diagnosis. Eur J Surg Oncol 2009;35:1146-51.

30 Badwe R, Parmar V, Hawaldar R, et al.: Surgical removal of primary tumor and axillary lymphnodes in women with metastatic breast cancer at first presentation: a randomized controlled trial. SABCS 2013; abstr S2-02.
31 Soran A, Ozmen V, Ozbas S, et al.: Early follow up of a randomized trial evaluating resection of the primary breast tumor in women presenting with de novo stage IV breast cancer; Turkish study (protocol MF07-01). SABCS 2013; abstr S2-03.

32 Khan SA: Surgery for the intact primary and stage IV breast cancer...lacking 'robust evidence'. Ann Surg Oncol 2013;20:2803-5.

33 Ruiterkamp J, Voogd AC, Tjan-Heijnen VC, et al.: SUBMIT: systemic therapy with or without upfront surgery of the primary tumor in breast cancer patients with distant metastases at initial presentation. BMC Surg 2012;12:5.

34 Dellas K: Does radiotherapy have curative potential in metastatic patients? The concept of local therapy in oligometastatic breast cancer. Breast Care 2011;6:363-8.

35 Tree AC, Khoo VS, Eeles RA, et al.: Stereotactic body radiotherapy for oligometastases. Lancet Oncol 2013;14:e28-37.

-36 Potters L, Kavanagh B, Galvin JM, et al.: American Society for Therapeutic Radiology and Oncology (ASTRO) and American College of Radiology (ACR) practice guideline for the performance of stereotactic body radiation therapy. Int $\mathrm{J}$ Radiat Oncol Biol Phys 2010;76:326-32.

37 Garcia-Barros M, Paris F, Cordon-Cardo C, et al.: Tumor response to radiotherapy regulated by endothelial cell apoptosis. Science 2003;300:1155-9.

38 Ben-Josef E, Lawrence TS: Using a bigger hammer: the role of stereotactic body radiotherapy in the management of oligometastases. J Clin Oncol 2009;27:1537-9.

39 Corbin KS, Hellman S, Weichselbaum RR: Extracranial oligometastases: a subset of metastases curable with stereotactic radiotherapy. J Clin Oncol 2013;31:1384-90.

40 Milano MT, Katz AW, Zhang H, Okunieff P: Oligometastases treated with stereotactic body radiotherapy: long-term follow-up of prospective study. Int J Radiat Oncol Biol Phys 2012;83:87886.

41 Chia SK, Speers CH, D'yachkova Y, et al.: The impact of new chemotherapeutic and hormone agents on survival in a population-based cohort of women with metastatic breast cancer. Cancer 2007;110: 973-9.

42 Rahman ZU, Frye DK, Smith TL, et al.: Results and long term follow-up for 1581 patients with metastatic carcinoma treated with standard dose doxorubicin-containing chemotherapy. Cancer 1999;85: 104-11.

43 Van Walsum GA, de Ridder JA, Verhoef C, et al.: Resection of liver metastases in patients with breast cancer: survival and prognostic factors. Eur J Surg Oncol 2012;38:910-7. 
44 Duan XF, Dong NN, Zhang T, Li Q: The prognostic analysis of clinical breast cancer subtypes among patients with liver metastases from breast cancer. Int J Clin Oncol 2013;18:26-32.

45 Atalya G, Biganzoli L, Renard F, et al.: Clinical outcome of breast cancer patients with liver metastases alone in the anthracycline taxane era: a retrospective analysis of two prospective, randomised metastatic breast cancer trials. Eur J Cancer 2003; 39:2439-49.

46 Chua TC, Saxena A, Liauw W, et al.: Hepatic resection for metastatic breast cancer: a systematic review. Eur J Cancer 2011;47:2282-90.

47 Howlader M, Heaton N, Rela M: Resection of liver metastases from breast cancer: towards a management guideline. Int J Surg 2011;9:285-91.

48 Abbott DE, Brouquet A, Mittendorf EA, et al.: Resection of liver metastases from breast cancer: estrogen receptor status and response to chemotherapy before metastasectomy define outcome Surgery 2012;151:710-6.

49 Cho SW, Kitisin K, Buck D, et al.: Transcatheter arterial chemoembolization is a feasible palliative locoregional therapy for breast cancer liver metastases. Int J Surg Oncol 2010;2010:251621.

50 Camacho LH, Kurzrock R, Cheung A: Pilot study of regional, hepatic intra-arterial paclitaxel in patients with breast carcinoma metastatic to the liver. Cancer 2007:109:2190-6.

51 Duan XF, Dong NN, Zhang T, Li Q: Treatment outcome of patients with liver-only metastases from breast cancer after mastectomy: a retrospective analysis. J Cancer Res Clin Oncol 2011;137: 1363-70.

52 Shah DR, Green S, Elliot A, et al.: Current oncologic applications of radiofrequency ablation therapies. J Gastrointest Oncol 2013;5:71-80.

53 Illing R, Gillams A: Radiofrequency ablation in the treatment of breast cancer liver metastases. Clin Oncol (R Coll Radiol) 2010;22:781-4.

-54 Taşçi Y, Aksoy E, Taşkın HE, et al.: A comparison of laparoscopic radiofrequency ablation versus systemic therapy alone in the treatment of breast cancer metastasis to the liver. HPB (Oxford) 2013;15:789-93.

55 Vogl TJ, Farshid P, Naguib NN, Zangos S: Thermal ablation therapies in patients with breast cancer liver metastases: a review. Eur Radiol 2013;23:797804.

56 Hoffmann RT, Paprottka P, Jakobs TF, et al.: Arterial therapies of non-colorectal cancer metastases to the liver (from chemoembolization to radioembolization). Abdom Imaging 2011;36:671-6.

57 Nichols FC: Pulmonary metastasectomy. Thorac Surg Clin 2012;22:91-9.

58 Rena O, Papalia E, Ruffini E, et al.: The role of surgery in the management of solitary pulmonary nodule in breast cancer patients. Eur J Surg Oncol 2007;33:546-50.

59 Diaz-Canton EA, Valero V, Rahman Z, et al.: Clinical course of breast cancer patients with metastases confined to the lungs treated with chemotherapy. Ann Oncol 1998;9:413-8.

60 Friedel G, Pastorino U, Ginsberg RJ, et al.: Results of lung metastasectomy from breast cancer: prognostic criteria on the basis of 467 cases of the International Registry of Lung Metastases. Eur J Cardiothorac Surg 2002;22:335-44.
61 Welter S, Jacobs J, Krbek T, et al.: Pulmonary metastases of breast cancer. When is resection indicated? Eur J Cardiothorac Surg 2008;34:1228-34.

62 Meimarakis G, Rüttinger D, Stemmler J, et al.: Prolonged overall survival after pulmonary metastasectomy in patients with breast cancer. Ann Thorac Surg 2013;95:1170-80.

63 Ibrahim T, Mercatali L, Amadori D: A new emergency in oncology: bone metastases in breast cancer patients. Oncol Lett 2013;6:306-10.

64 Husband JE, Padhani AR (eds): Metastatic Bone Tumours: Recommendations for Cross-Sectional Imaging in Cancer Management. London, The Royal College of Radiologists, 2006, pp. 108-10.

65 Solomayer EF, Diel IJ, Meyberg, et al.: Metastatic breast cancer: clinical course, prognosis and therapy related to the first site of metastasis. Breast Cancer Res Treat 2000;59:271-8.

66 Goldhirsch A, Gelber RD, Castiglione M: Relapse of breast cancer after adjuvant treatment in premenopausal and perimenopausal women: patterns and prognoses. J Clin Oncol 1988;6:89-97.

67 Ahn SG, Lee HM, et al.: Prognostic factors for patients with bone-only metastasis in breast cancer. Yonsei Med J 2013;54:1168-77.

68 Domchek SM, Younger J, Finkelstein DM, Seiden MV: Predictors of skeletal complications in patients with metastatic breast carcinoma. Cancer 2000;89:363-8.

69 Koizumi M, Yoshimoto M, Kasumi F, Ogata E: Comparison between solitary and multiple skeletal metastatic lesions of breast cancer patients. Ann Oncol 2003;14:1234-40.

70 Wedin R 2001, Bauer HC, Rutqvist LE: Surgical treatment for skeletal breast cancer metastases: a population-based study of 641 patients. Cancer 2001;92:257-62.

71 Wegener B, Schlemmer M, Stemmler J, et al.: Analysis of orthopedic surgery of bone metastases in breast cancer patients. BMC Musculoskelet Disord 2012;13:232

72 Milano MT, Katz AW, Okunieff P: Patterns of recurrence after curative-intent radiation for oligometastases confined to one organ. Am J Clin Oncol 2010;33:157-63.

73 Yimin Y, Zhiwei R, Wei M, Jha R: Current status of percutaneous vertebroplasty and percutaneous kyphoplasty - a review. Med Sci Monit 2013;19: 826-36.

74 Trumm CG, Jakobs TF, Zech CJ, et al.: CT fluoroscopy-guided percutaneous vertebroplasty for the treatment of osteolytic breast cancer metastases: results in 62 sessions with 86 vertebrae treated. J Vasc Interv Radiol 2008;19:1596-606.

75 Dawood S, Gonzalez-Angulo AM: Progress in the biological understanding and management of breast cancer-associated central nervous system metastases. Oncologist 2013;18:675-84.

76 Lim E, Lin NU: New insights and emerging therapies for breast cancer brain metastases. Oncology 2012;26:652-9, 663.

77 Berghoff A, Bago-Horvath Z, De Vries C, et al.: Brain metastases free survival differs between breast cancer subtypes. Br J Cancer 2012;106: 440-6.

78 Weil RJ, Palmieri DC, Bronder JL, et al.: Breast cancer metastasis to the central nervous system. Am J Pathol 2005;167:913-20.
79 Berghoff AS, Bago-Horvath Z, Ilhan-Mutlu A, et al.: Brain-only metastatic breast cancer is a distinct clinical entity characterised by favourable median overall survival time and a high rate of long-term survivors. Br J Cancer 2012;107:1454-8.

80 Niwińska A, Murawska M: New breast cancer recursive partitioning analysis prognostic index in patients with newly diagnosed brain metastases. Int J Radiat Oncol Biol Phys 2012;82:2065-71.

81 Anders CK, Deal AM, Miller CR, et al.: The prognostic contribution of clinical breast cancer subtype, age, and race among patients with breast cancer brain metastases. Cancer 2011;117:1602-11.

82 Dyer MA, Kelly PJ, Chen YH, et al.: Importance of extracranial disease status and tumor subtype for patients undergoing radiosurgery for breast cancer brain metastases. Int J Radiat Oncol Biol Phys 2012;83:e479-86.

83 Aoyama H, Shirato H, Tago M, et al.: Stereotactic radiosurgery plus whole-brain radiation therapy vs stereotactic radiosurgery alone for treatment of brain metastases: a randomized controlled trial. JAMA 2006;295:2483-91.

84 Kocher M, Soffietti R, Abacioglu U, et al.: Adjuvant whole brain radiotherapy versus observation after radiosurgery or surgical resection of 1 to 3 cerebral metastases: results of the EORTC 2295226001 study. J Clin Oncol 2011;29:134-41.

85 Linskey ME, Andrews DW, Asher AL, et al.: The role of stereotactic radiosurgery in the management of newly diagnosed brain metastases: a systematic review and evidence-based clinical practice guideline. J Neurooncol 2010;96:45-68.

86 Gaspar LE, Mehta MP, Patchell RA, et al.: The role of whole brain radiation therapy in the management of newly diagnosed brain metastases: a systematic review and evidence-based clinical practice guideline. J Neurooncol 2010;96:17-32.

87 Kalkanis SN, Kondziolka D, Gaspar LE, et al.: The role of surgical resection in the management of newly diagnosed brain metastases: a systematic review and evidence-based clinical practice guideline. J Neurooncol 2010;96:33-43.

88 Patil CG, Pricola K, Sarmiento JM, et al.: Whole brain radiation therapy (WBRT) alone versus WBRT and radiosurgery for the treatment of brain metastases. Cochrane Database Syst Rev 2012;9: CD006121.

89 Andrews DW, Scott CB, Sperduto PW, et al.: Whole brain radiation therapy with or without stereotactic radiosurgery boost for patients with one to three brain metastases: phase III results of the RTOG 9508 randomised trial. Lancet 2004;363: 1665-72.

90 Combs SE, Schulz-Ertner D, et al.: Treatment of cerebral metastases from breast cancer with stereotactic radiosurgery. Strahlenther Onkol 2004;180: 590-6.

91 Chargari C, Campana F, Pierga JY, et al.: Wholebrain radiation therapy in breast cancer patients with brain metastases. Nat Rev Clin Oncol 2010;7: 632-40.

92 Caballero JA, Sneed PK, Lamborn KR, et al.: Prognostic factors for survival in patients treated with stereotactic radiosurgery for recurrent brain metastases after prior whole brain radiotherapy. Int J Radiat Oncol Biol Phys 2012;83:303-9.

93 Kelly PJ, Lin NU, Claus EB, et al.: Salvage stereotactic radiosurgery for breast cancer brain metastases: outcomes and prognostic factors. Cancer 2012; 118:2014-20. 